ARTICLE

\title{
Ultrastable microwave and soliton-pulse generation from fibre-photonic-stabilized microcombs
}

\author{
Dohyeon Kwon ${ }^{1,4}$, Dongin Jeong ${ }^{2,4}$, Igju Jeon ${ }^{1}$, Hansuek Lee (i] ${ }^{2,3 凶}$ \& Jungwon Kim (i] ${ }^{1 凶}$
}

The ability to generate lower-noise microwaves has greatly advanced high-speed, highprecision scientific and engineering fields. Microcombs have high potential for generating such low-noise microwaves from chip-scale devices. To realize an ultralow-noise performance over a wider Fourier frequency range and longer time scale, which is required for many high-precision applications, free-running microcombs must be locked to more stable reference sources. However, ultrastable reference sources, particularly optical cavity-based methods, are generally bulky, alignment-sensitive and expensive, and therefore forfeit the benefits of using chip-scale microcombs. Here, we realize compact and low-phase-noise microwave and soliton pulse generation by combining a silica-microcomb (with few-mm diameter) with a fibre-photonic-based timing reference (with few-cm diameter). An ultrastable $22-\mathrm{GHz}$ microwave is generated with $-110 \mathrm{dBc} / \mathrm{Hz}(-88 \mathrm{dBc} / \mathrm{Hz})$ phase noise at $1-\mathrm{kHz}(100-\mathrm{Hz})$ Fourier frequency and $10^{-13}$-level frequency instability within 1-s. This work shows the potential of fully packaged, palm-sized or smaller systems for generating both ultrastable soliton pulse trains and microwaves, thereby facilitating a wide range of field applications involving ultrahigh-stability microcombs.

\footnotetext{
${ }^{1}$ School of Mechanical and Aerospace Engineering, Korea Advanced Institute of Science and Technology (KAIST), Daejeon 34141, Korea. ${ }^{2}$ Graduate Schoo of Nanoscience and Technology, Korea Advanced Institute of Science and Technology (KAIST), Daejeon 34141, Korea. ${ }^{3}$ Department of Physics, Korea Advanced Institute of Science and Technology (KAIST), Daejeon 34141, Korea. ${ }^{4}$ These authors contributed equally: Dohyeon Kwon, Dongin Jeong.

凶email: hansuek@kaist.ac.kr; jungwon.kim@kaist.ac.kr
} 
T he generation of lower-noise and higher-stability microwaves has advanced a wide range of fields in science and engineering ranging from radio astronomy and particle accelerators to radars and telecommunications. Various photonic methods have been developed to generate such low-noise microwaves in the last three decades ${ }^{1-9}$, for example, optoelectronic oscillators $(\mathrm{OEOs})^{1,2}$, electro-optical frequency division ${ }^{3-5}$ and monolithic mode-locked lasers ${ }^{6}$. In particular, by coherently linking the optical and microwave frequency domains, the use of optical frequency combs has become a powerful method for producing low-noise and ultrastable microwave signals ${ }^{7-10}$. The optical frequency division (OFD) of an ultrastable continuouswave $(\mathrm{CW})$ light by a mode-locked fibre frequency comb has enabled the generation of $-130 \mathrm{dBc} / \mathrm{Hz}$-level microwaves at 10 $\mathrm{Hz}$ Fourier frequency ${ }^{10}$. In addition to low-noise microwave generation, frequency combs and photonic methods are also highly effective at transferring low-noise and synchronised microwave signals and optical pulse trains to remote locations for newly emerging ultrahigh-precision applications, for example, the synchronisation of large-scale X-ray science facilities ${ }^{11-13}$ and the distribution of phase-synchronised microwaves for very long baseline interferometry (VLBI)-based radio astronomy ${ }^{14,15}$.

More recently, microresonator-based optical frequency combs (microcombs), different from traditional mode-locked oscillatorbased combs, have been intensively studied and rapidly developed ${ }^{16-22}$. Microcombs generate soliton optical pulse trains using a nonlinear frequency conversion process of parametric four-wave mixing (FWM) in a microresonator. The main advantages of microcombs, in contrast to mode-locked oscillator combs, are their compact size and high repetition rate. Microcombs can generate soliton-pulse trains with $\sim 10 \mathrm{GHz}$ to $\sim 1 \mathrm{THz}$ repetition rate from tens-of-micrometre to few-millimetre-sized chips. Further, the quantum-limited fast timing jitter of freerunning microcombs ${ }^{23}$ was reported to be on the order of only a few femtoseconds 24,25 , the regime of which is difficult to achieve with electronic oscillators. These features make microcombs an ideal comb source for various microwave photonic applications ${ }^{26-30}$. Indeed, recent works have shown the potential for microcomb-based microwave generation ${ }^{31-36}$. For example, a free-running $14-\mathrm{GHz} \mathrm{MgF}_{2}$ microcomb reported the phase noise of $-115 \mathrm{dBc} / \mathrm{Hz}(-80 \mathrm{dBc} / \mathrm{Hz})$ at $1-\mathrm{kHz}(100-\mathrm{Hz})$ Fourier frequency ${ }^{32}$ and a free-running $20-\mathrm{GHz}$ silicon-nitride microcomb demonstrated the phase noise of $-80 \mathrm{dBc} / \mathrm{Hz}(-45 \mathrm{dBc} /$ $\mathrm{Hz})$ at $1-\mathrm{kHz}(100-\mathrm{Hz})$ Fourier frequency ${ }^{33}$.

Although free-running microcombs have demonstrated the potential to reach a previously inaccessible regime of ultralow timing jitter and microwave phase noise with repetition rates extending to tens of gigahertz, timing jitter and repetition-rate phase noise must be suppressed over a longer time scale (down to lower Fourier frequency range) to satisfy a wide range of highprecision applications. Accordingly, several previous works have attempted to stabilise the microwave repetition rate of microcombs, including microwave phase-locking using frequency synthesisers $32-35,37,38$ and ultrastable cavity-locked pump lasers ${ }^{34,39}$. However, the lack of a compact, mechanically robust, broadband and high-performance microcomb stabilisation method has been a major bottleneck in achieving the field applications of ultrastable and compact microcombs in microwave photonic systems.

Here, we demonstrate the generation of low-phase-noise K-band microwaves from a palm-sized photonic platform by combining a silica microcomb and a fibre-photonic timing stabilisation method. To stabilise the timing, a kilometres-long fibre delayline, wound as a compact spool with a several-cm outer diameter, which was previously used for stabilising mode-locked Er-fibre combs ${ }^{40}$, was used as a timing reference. In order to extend the repetition-rate locking bandwidth over $>100 \mathrm{kHz}$, we also developed a new high-speed repetition-rate tuning mechanism using a customised voltage-controlled oscillator (VCO) and an acousto-optic frequency shifter (AOFS), which can be also applied for other types of ultrahigh- $Q$ resonator-based combs. In addition to the compact size of the platform, this stabilisation method operates at room temperature, does not require a vacuum environment and is completely alignment-free, making it ideal for use with ultracompact microcombs. By employing the fibre-photonic stabilisation, the $22-\mathrm{GHz}$ microwave phase noise generated from a free-running silica microcomb $(-30 \mathrm{dBc} / \mathrm{Hz}$ at $10 \mathrm{~Hz}$ Fourier frequency) could be suppressed by $>40 \mathrm{~dB}$ down to $-74 \mathrm{dBc} / \mathrm{Hz}$. The resulting integrated timing jitter of the $22-\mathrm{GHz}$ microwave signal is only $10.4 \mathrm{fs}$ when integrated from $10 \mathrm{~Hz}$ to $1 \mathrm{MHz}$, which corresponds to the $10^{-13}$. level frequency instability within 1-s. With its excellent phase noise, stability, compactness and robustness, the demonstrated system can be readily applied in various field applications, including radio astronomy, photonics-based radars ${ }^{41}, 5 / 6 \mathrm{G}$ telecommunications and signal analysis instruments.

\section{Results}

System overview. The principle of ultrastable microwave and soliton-pulse generation from a timing-stabilised silica microcomb is shown in Fig. 1a. A pulse train with $22-\mathrm{GHz}$ repetition rate is generated via FWM and soliton mode-locking processes in a silica microresonator with a 3-mm-diameter driven by a $\mathrm{CW}$ pump laser. A $22-\mathrm{GHz}$ microwave signal is extracted from an optical pulse train by an optical-to-electronic (OE) conversion process using a modified uni-travelling carrier (MUTC) photodiode followed by a radio-frequency (RF) bandpass filter and an $\mathrm{RF}$ amplifier. Since the timing jitter and repetition-rate phase noise from a free-running microcomb diverge over time, the repetition rate is stabilised by a fibre-photonic stabiliser ${ }^{42}$, which obtains signals containing the frequency noise of two comb modes (i.e. $v_{m}=m f_{\text {rep }}+f_{\text {ceo }}$ and $v_{n}=n f_{\text {rep }}+f_{\text {ceo }}$, where $f_{\text {rep }}$ is the repetition rate, $f_{\text {ceo }}$ is the carrier-envelope offset frequency and $m$ and $\mathrm{n}$ are mode numbers; see Fig. 1b). The frequency noise difference between two comb modes is extracted by mixing the signals, which corresponds to the frequency noise of $(m-n) f_{\text {rep }}$. Once the feedback loop is closed, the stability of the microcomb repetition rate $\left(\delta f_{\text {rep }} / f_{\text {rep }}\right)$ can reach up to the stability of the time delay of the fibre link $(\delta \tau / \tau)$.

Soliton microcomb. A silica wedge microresonator is used for the soliton generation. The free spectral range $\left(D_{1} / 2 \pi\right)$ of a $3-\mathrm{mm}$ diameter resonator is $\sim 22 \mathrm{GHz}$. The intrinsic $\mathrm{Q}$ factor of the resonator is $2.5 \times 10^{8}$ and the second-order dispersion $\left(D_{2} / 2 \pi\right)$ is $13.3 \mathrm{kHz}$. A pump laser at $1550 \mathrm{~nm}$ is amplified by an Er-doped fibre amplifier (EDFA; EDFA 1 in Fig. 1a) and the amplified spontaneous emission (ASE) noise is removed by a bandpass filter. The pump laser is coupled into and out of the resonator using a tapered fibre after the polarisation is adjusted to the microresonator mode. A rapid scan of the pump frequency from blue-to-red detuning allows soliton generation. To avoid thermal destabilization of the soliton, the pump-cavity detuning is locked via a piezoelectric transducer (PZT) of the pump laser ${ }^{43,44}$. An acousto-optic frequency shifter (AOFS; AOFS $_{1}$ in Fig. 1a) mitigates thermal destabilization by implementing rapid power modulation within a few microseconds when a soliton is generated and allows frequency modulation to stabilise the repetition rate (see Methods and Supplementary Information). The residual pump signal is removed by a notch filter and the soliton is amplified by an EDFA (EDFA ${ }_{2}$ in Fig. 1a) for microwave generation and comb stabilisation. From the amplified power, 90 


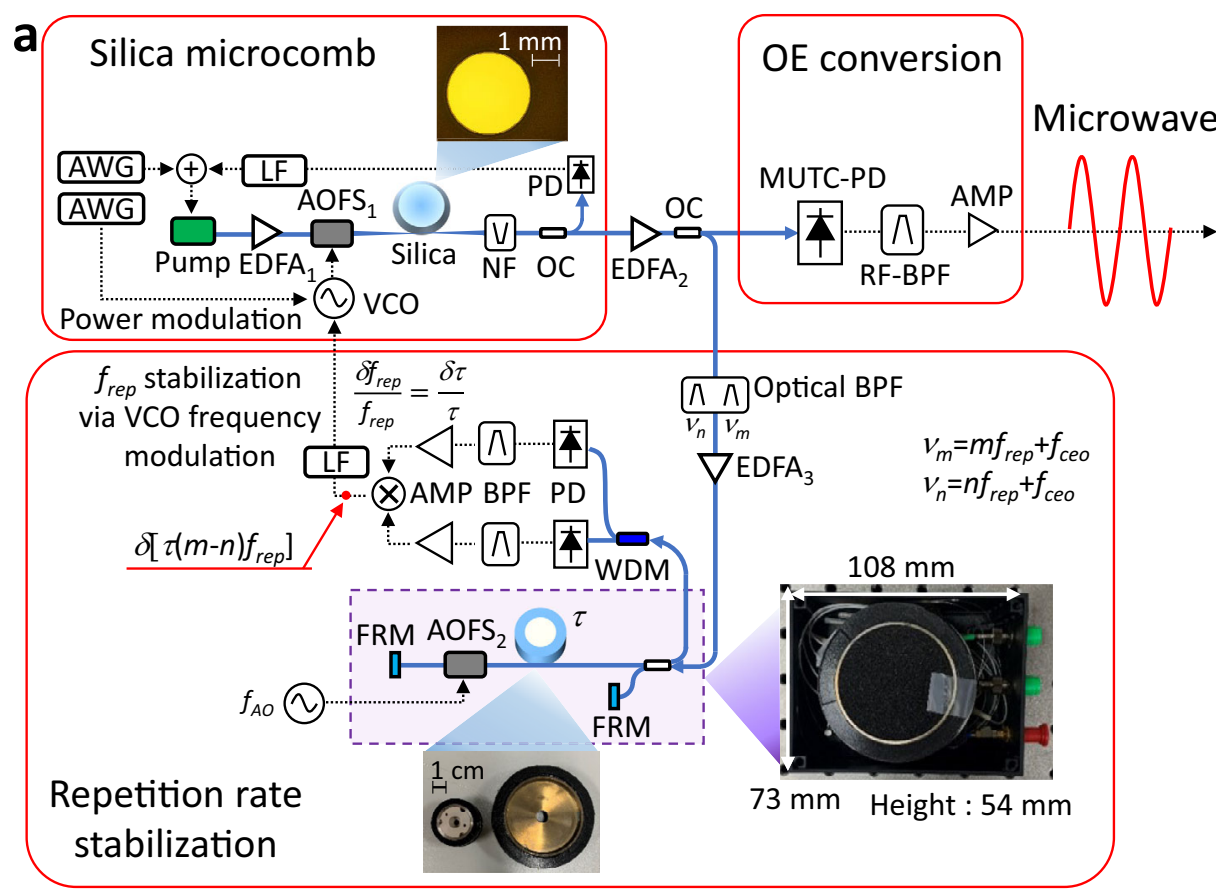

b

(i) Free-running soliton

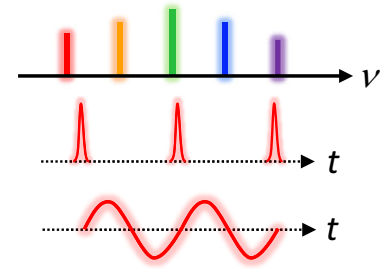

(ii) Fibre-stabilized soliton

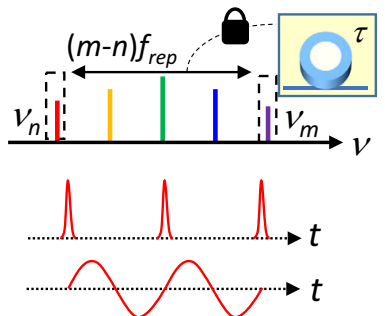

Fig. 1 Ultrastable microwave generation from a silica microcomb stabilised by fibre photonics. a Schematic of ultrastable microwave generation from a fibre-stabilised silica microcomb. AWG arbitrary waveform generator, EDFA erbium-doped fibre amplifier, AOFS acousto-optic frequency shifter, VCO voltage-controlled oscillator, NF notch filter, OC optical coupler, PD photodiode, LF loop filter, MUTC-PD modified uni-travelling carrier photodiode, RF-BPF 250-MHz-bandwidth radio-frequency bandpass filter at 22-GHz, AMP RF amplifier, BPF RF-bandpass filter at $2 f_{A O}$, Optical BPF bandpass filter for $\nu_{n}$ and $\nu_{m}, f_{\text {rep }}$ repetition rate, $f_{\text {ceo }}$ carrier-envelope offset frequency, $m$ and $n$ mode number of $\nu_{m}$ and $\nu_{n}$, respectively, FRM Faraday rotating mirror, $f_{A O}$ driving frequency for $\mathrm{AOFS}_{2}, \tau$ time delay induced by the fibre link. An optical microscope image of a silica microresonator is shown at the top of the figure. A photograph of a 200-m-long fibre link (left) and a 1-km-long fibre link (right) is displayed at the bottom of the figure. A photograph of the entire fibrephotonic repetition-rate stabiliser (with a case size of $108 \mathrm{~mm} \times 73 \mathrm{~mm} \times 54 \mathrm{~mm}$ ) is shown on the right side of the figure. $\mathbf{b}$ Concept of comb-mode frequency noise, timing jitter, microwave phase noise of a free-running microcomb (i) and a fibre-stabilised microcomb (ii).
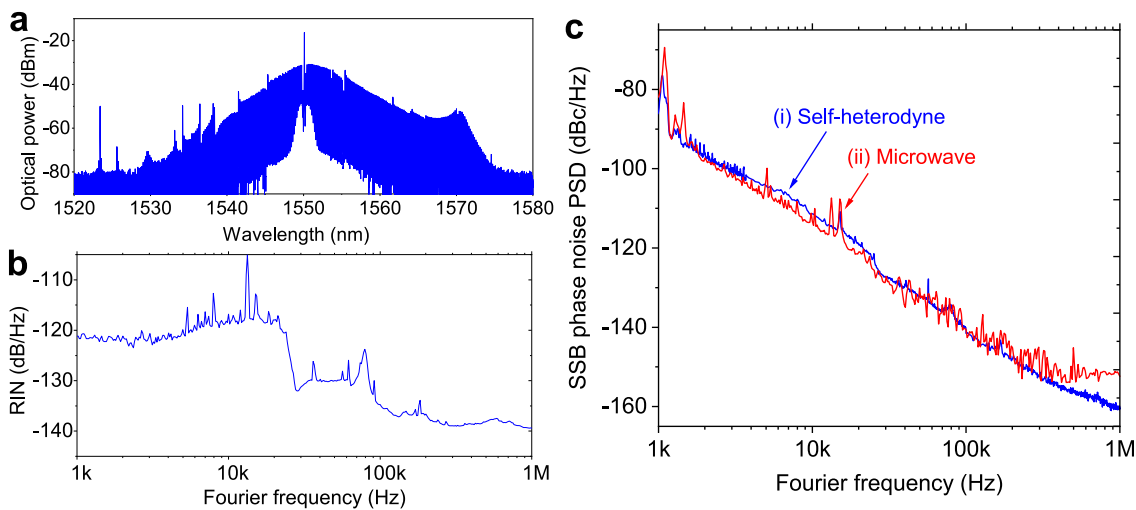

Fig. 2 Properties of the free-running silica microcomb. a Optical spectrum of the silica microcomb. $\mathbf{b}$ Relative intensity noise (RIN) of the microcomb. Note that the sudden jump at $20 \mathrm{kHz}$ is due to the response of the PZT in the pump laser. c Repetition-rate phase noise of the free-running microcomb measured in the (i) optical domain and (ii) microwave domain. Source data for Fig. 2a, b and c are provided in the Supplementary Information.

percent of the amplified power is used to generate microwaves, while 10 percent of the optical power is tapped to stabilise the repetition rate.

The typical performance of the soliton is shown in Fig. 2. The estimated pulsewidth from the $\operatorname{sech}^{2}$-shaped optical spectrum of the microcomb (Fig. 2a) is 350 fs. The intensity fluctuation of the pulse train is measured as the relative intensity noise (RIN), as shown in Fig. 2b. The single-sideband (SSB) repetition-rate phase-noise power spectral density (PSD) of the free-running microcomb (curve (i) in Fig. 2c) is first measured in the optical domain by a fibre delayline-based self-heterodyne method 24,45 (see Supplementary Fig. 10). Due to the high-resolution of the self-heterodyne measurement method, the repetition-rate phase noise is accurately characterised below $-160 \mathrm{dBc} / \mathrm{Hz}$ at $1-\mathrm{MHz}$ Fourier frequency. We also measured the SSB phase noise of the extracted $22-\mathrm{GHz}$ microwave signal (curve (ii) in Fig. 2c) using a commercial phase noise analyser (Keysight, E5052B + E5053A), which shows a good agreement with the optical-domain measurement result (curve (i) in Fig. 2c). The slight difference in the $>500-\mathrm{kHz}$ Fourier frequency range is due to the photodetection white noise floor and the measurement sensitivity of the phase noise analyser.

The repetition-rate phase noise of the free-running comb can be minimised by adjusting the pump-cavity frequency detuning 
to the quiet point ${ }^{31,33}$ (see Supplementary Fig. 3). In our system, the phase noise is minimised when the pump-cavity detuning $(\delta \omega)$ is $16 \mathrm{MHz}$ according to the relation $\delta \omega=\left(D_{2} / 2 D_{1}^{2}\right)\left(1 / \tau_{p}^{2}\right)$, where $\tau_{p}$ is the pulsewidth ${ }^{31}$. The measured phase noise at $10-$ $\mathrm{kHz}(100-\mathrm{kHz})$ Fourier frequency is $-111 \mathrm{dBc} / \mathrm{Hz}(-141 \mathrm{dBc} /$ $\mathrm{Hz}$ ) with an integrated timing jitter of $2.14 \mathrm{fs}$ (249 as) when integrated from $10-\mathrm{kHz}(100-\mathrm{kHz})$ to $1-\mathrm{MHz}$ Fourier frequency range. Compared to the recently demonstrated free-running 20 $\mathrm{GHz}$ silicon-nitride microcomb $\mathrm{b}^{33}$, the phase noise of the demonstrated silica microcomb is more than $10 \mathrm{~dB}$ lower at $100-\mathrm{kHz}$ Fourier frequency due to the material properties of the silica microresonator $23,31,46$.

Note that we conducted the repetition-rate stabilisation where the pump-cavity detuning is $\sim 500-\mathrm{kHz}$ blue side to the quiet point, where the lowest phase noise of the stabilised microcomb is achieved. We believe that, at the exact quiet point, the comb is the least sensitive to the pump disturbance (i.e. $\delta f_{\text {rep }} / \delta v_{\text {pump }} \sim 0$ ) and has a difficulty in increasing the loop gain when stabilising ${ }^{34}$. We found that the repetition-rate phase noise (timing jitter) could be suppressed most at a slightly blue-detuned position from the exact quiet point, where the free-running jitter is still low and the pump sensitivity is high enough for effectively increasing the loop gain of the feedback control loop.

Microcomb repetition-rate stabilisation. The working principle of low-phase-noise microwave generation is based on repetitionrate stabilisation using a compact fibre Michelson interferometer, as illustrated in Fig. 1a (also see Supplementary Figs. 10 and 11). Two frequency modes (i.e. $v_{m}=m f_{\text {rep }}+f_{\text {ceo }}$ and $v_{n}=n f_{\text {rep }}+f_{\text {ceo }}$ ) are filtered by fibre-Bragg gratings to extract repetition-rate frequency noise. A fibre link is used as both the timing reference and the frequency noise discriminator. In this work, we selected a 1$\mathrm{km}$-long (2-km-long round-trip) fibre link by considering both detection sensitivity (scaling with delay time $\tau$ ) and detection bandwidth (scaling with inverse of delay time, $1 / \tau$ ). Note that, depending on the intended performance and application, different length of fibre link can be also used (see Supplementary Fig. 8 for comparison of stabilised phase noise with different delay length). The fibre link is wound with few-centimetre diameter (e.g. $7 \mathrm{~cm}$ diameter for 1-km-long fibre) using the fibre-winding method used for making fibre-optic gyroscopes. An AOFS driven by $f_{A O}\left(\mathrm{AOFS}_{2}\right.$ in Fig. 1a) is inserted at the delay arm to reduce background noise by synchronous detection. The absolute frequency noise of each comb mode by optical carrier interference is photodetected individually using a wavelength division multiplexing (WDM) coupler. The carrier frequency of each photodetected signal is $2 f_{\mathrm{AO}}$ induced by the round-trip frequency shift due to $\mathrm{AOFS}_{2}$ in the delay arm. The phase noise of $2 f_{\mathrm{AO}}$ contains the absolute frequency noise of each corresponding comb mode weighted by the time delay (i.e. $\delta\left[\tau \times\left(m f_{\text {rep }}+f_{\text {ceo }}+2 f_{\mathrm{AO}}\right)\right]$ and $\left.\delta\left[\tau \times\left(n f_{\text {rep }}+f_{\text {ceo }}+2 f_{\mathrm{AO}}\right)\right]\right)$. Each photodetected signal is bandpass-filtered at $2 f_{\mathrm{AO}}$ and mixed by a frequency mixer, which results in the rejection of common-mode $f_{\text {ceo }}$ and $2 f_{\mathrm{AO}}$ noise. The resulting lowpass-filtered baseband mixer output, therefore, contains only the repetition-rate frequency noise (i.e. $\left.\delta\left[\tau \times(m-n) f_{\text {rep }}\right]\right)$.

While mode-locked fibre combs could be easily locked to the fibre reference by intra-cavity PZTs, locking the microcombs, ultrahigh-Q resonator-based combs such as silica microcombs in particular, is not straightforward due to the lack of a direct mechanical actuator inside the microresonator. In particular, the previous approaches using pump power modulation could achieve only a few $\mathrm{kHz}$ bandwidth ${ }^{47}$, which is too narrowband for effective repetition-rate stabilisation. In this work, we employed the AOFS for broadband locking of silica microcombs to the fibre reference. Since the repetition-rate frequency noise is coupled to the residual cavity-pump detuning noise ${ }^{31}$, it can be suppressed via feedback control to the voltage-controlled oscillator (VCO) applied to the AOFS before the silica microresonator $\left(\mathrm{AOFS}_{1}\right.$ in Fig. 1a). Here, the frequency modulation-based stabilisation can provide a much larger control bandwidth than pump power modulation: the 3-dB control bandwidth of the demonstrated system can reach $\sim 120 \mathrm{kHz}$ as shown on the curve (ii) in Fig. 3a. Note that this control bandwidth was limited by the fibre delay time itself (which is set by $1 / \tau$ ), and the actuation bandwidth itself achieved by the AOFS and the customised VCO is much larger, over $400 \mathrm{kHz}$. Also note that, while this broadband repetition-rate tuning mechanism is
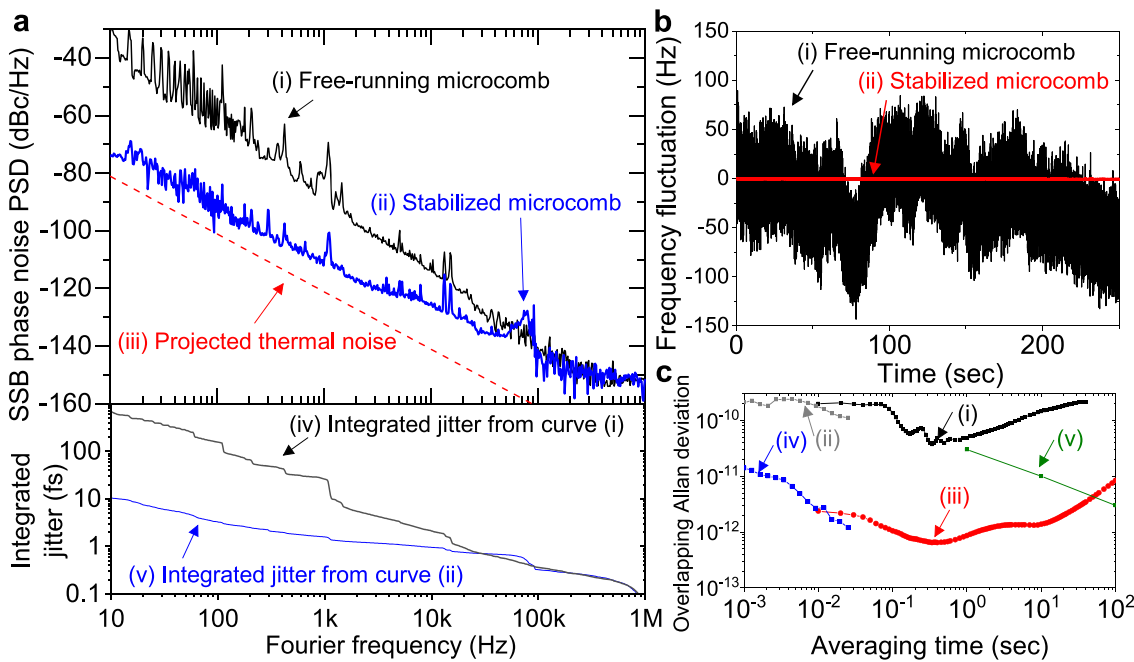

Fig. 3 Characterisation of the fibre-stabilised silica microcomb. a 22-GHz microwave phase noise and integrated timing jitter. (i) Phase noise of the freerunning microcomb. (ii) Phase noise of the 1- $\mathrm{km}$ fibre-stabilised microcomb. (iii) Projected thermal noise-limited phase noise ${ }^{53}$ of the 1-km fibre-photonic stabiliser. (iv) Integrated root-mean-square timing jitter of the free-running microcomb. (v) Integrated root-mean-square timing jitter from the fibrestabilised microcomb. b Long-term frequency fluctuation of the repetition rate. (i) Free-running microcomb (black). (ii) Fibre-stabilised microcomb (red). c Repetition-rate frequency instability of the microcomb. (i) Free-running microcomb (computed from curve (i) in Fig. 3b). (ii) Free-running microcomb (computed from curve (i) in Fig. 3a). (iii) Fibre-stabilised microcomb (computed from curve (ii) in Fig. 3b). (iv) Fibre-stabilised microcomb (computed from curve (ii) in Fig. 3a). (v) Chip-scale atomic reference (SA55, Microsemi). Source data for Fig. 3a, b and c are provided in the Supplementary Information. 
applied for a silica microcomb and a fibre delayline-based reference source in our work, it can be also used for other types of ultrahigh-Q microresonators and reference sources for broadband stabilisation of microcomb repetition rates. We also confirmed that the crosstalk between the repetition-rate stabilisation via frequency modulation of the VCO and the soliton modelocking via the pump laser PZT is negligible, and both controls can be used simultaneously (see Supplementary Information and Supplementary Fig. 7).

For the demonstrated system, a fibre Michelson interferometer using a 1-km-long fibre link with a diameter of $7 \mathrm{~cm}$ was packaged in a $108 \mathrm{~mm} \times 73 \mathrm{~mm} \times 54 \mathrm{~mm}$-sized, air-tight box (see Fig. 1a). Note that, the present-day size of the fibre-photonic stabiliser is mostly limited by the size of $\mathrm{AOFS}_{2}$ due to the required space for the frequency-shifted diffracted beam inside an AOFS device. The system size can be further reduced by using an SSB phase modulator 47,48 instead of an AOFS. In addition, fibre-optic components can be compactly integrated on a single photonic integrated circuit (PIC) assisted by silicon photonics ${ }^{26,49-51}$.

OE conversion. To avoid a pulsewidth-dependent noise floor at high offset frequencies ${ }^{52}$, the soliton pulsewidth is compressed to $<1$ ps using a dispersion compensating fibre. More than $15 \mathrm{~mW}$ of optical power is applied to an MUTC photodiode. The generated photocurrent with a $-8 \mathrm{~V}$ bias voltage is $\sim 7 \mathrm{~mA}$. The generated microwave is filtered by a bandpass filter centred at $22 \mathrm{GHz}$ and amplified by a low-phase-noise RF amplifier up to $+10 \mathrm{dBm}$.

Microwave phase noise and frequency stability performances. The phase noise of the $22-\mathrm{GHz}$ microwaves extracted from the silica microcomb is characterised by commercial phase noise analysers (Keysight, E5052B + E5053A). The SSB PSD of the 1$\mathrm{km}$-long fibre-stabilised microcomb is shown as curve (ii) in Fig. 3a. The phase noise PSD of the fibre-stabilised microcomb is $-110 \mathrm{dBc} / \mathrm{Hz}(-88 \mathrm{dBc} / \mathrm{Hz})$ at $1-\mathrm{kHz}(100-\mathrm{Hz})$ Fourier frequency. Compared to that of the free-running microcomb (curve (i) in Fig. 3a), the phase noise of the fibre-stabilised microcomb (curve (ii) in Fig. 3a) is suppressed by more than $30 \mathrm{~dB}$ for Fourier frequency of $<100 \mathrm{~Hz}$, which results in effective frequency stabilisation of the microcomb-generated microwave signal. Using the fibre stabiliser, the integrated root-mean-square (RMS) timing jitter is greatly suppressed from $689 \mathrm{fs}$ (curve (iv) in Fig. 3a) to $10.4 \mathrm{fs}$ (curve (v) in Fig. 3a) over the 0.1-s integration time. The timing jitter over 0.1 -ms time scale, which is important for telecommunications and analogue-to-digital data converters (ADCs), reaches even the subfemtosecond regime (0.96 fs) [integration bandwidth: $10 \mathrm{kHz}-1 \mathrm{MHz}$. Note that, in the high Fourier frequency $(>300 \mathrm{kHz})$, the measured photodetection noise floor $(\sim-153 \mathrm{dBc} / \mathrm{Hz})$ is degraded from the projected shot noise limit $(-161 \mathrm{dBc} / \mathrm{Hz})$ by the noise figure as well as the amplitude-to-phase conversion of the used RF amplifier.

The fundamental phase-noise limit of the fibre-photonic stabiliser originates from the thermomechanical and thermoconductive fibre-length fluctuation ${ }^{53}$ that destabilises the timing stability of the fibre delayline (curve (iii) in Fig. 3a, see Supplementary Information). There is a $\sim 10-\mathrm{dB}$ discrepancy between the stabilised phase noise and the fundamental limit; we believe that this discrepancy originates from the Rayleigh scattering-limited intensity noise to the frequency noise conversion $^{54}$ and the limited detection sensitivity of the fibre stabiliser, where the error signal, $\delta\left[\tau \times(m-n) f_{\text {rep }}\right]$, scales with the fibre delay time $\tau$ and the frequency separation between bandpass filters $(m-n) f_{\text {rep }}$ (see Supplementary Information).
The long-term stability is evaluated by the frequency instability computed from the frequency fluctuation. To measure the frequency fluctuation with high-sensitivity digital cross-correlation ${ }^{55}$, the $\mathrm{K}$-band microwave is down-converted to a low frequency. Two independent silica microcombs, each stabilised to an independent fibre-photonic stabiliser, are used for the down-conversion (see Supplementary Information and Supplementary Fig. 12). The fundamental repetition rates of the two microcombs are 22.059 and $22.083 \mathrm{GHz}$, and the down-converted microwave beat note of the two microcombs is $23.3 \mathrm{MHz}$. The frequency fluctuation of the microwave beat note is sampled by a frequency counter with a sampling rate of $100 \mathrm{~Hz}$. While the frequency fluctuation of the freerunning microcombs (curve (i) in Fig. 3b) is larger than $100 \mathrm{~Hz}$, the frequency fluctuation of the fibre-stabilised microcombs (curve (ii) in Fig. $3 \mathrm{~b}$ ) is less than $2 \mathrm{~Hz}$. The frequency instability evaluated by the overlapping Allan deviation is computed from the frequency fluctuation measurement (for $>10^{-2} \mathrm{~s}$ ) and the phase noise measurement ${ }^{56}$ (from $10^{-3} \mathrm{~s}$ to $10^{-2} \mathrm{~s}$ ). For the free-running microcombs (curves (i) and (ii) in Fig. 3c), the minimum frequency instability is on the level of $10^{-11}$ and diverges after $0.4 \mathrm{~s}$. For the fibre-stabilised microcombs (curves (iii) and (iv) in Fig. 3c), the frequency instability starts from $1.4 \times 10^{-11}$ at $10^{-3} \mathrm{~s}$ and reaches $4.6 \times 10^{-13}$ at $0.4 \mathrm{~s}$.

The frequency instability starts increasing after $0.4 \mathrm{~s}$ due to the time-dependent drift. For the free-running microcomb, we believe that the time-dependent timing drift at this time scale is due to the thermally-induced path length change of the silica material, which was also observed in silica-based cavity mirrors ${ }^{57}$. For the fibre-stabilised microcomb, the stability of the repetition rate follows the timing stability of the fibre link. The SMF-28 fibre has a thermal coefficient of delay (TCD) of $\sim 40-130 \mathrm{ps} / \mathrm{km} /$ $\mathrm{K}^{58,59}$, and this time-dependent temperature drift in the fibre link induces Allan deviation diverging after $\sim 0.4 \mathrm{~s}$ (see Supplementary Information). To ensure longer-term stable operation while retaining the compact overall size, chip-scale atomic references can be additionally used. For example, one of the best chip-scale atomic references (curve (v) in Fig. 3c, SA55 from Microsemi) can improve the performance of our system after $40 \mathrm{~s}$, where the frequency instability of the atomic reference becomes lower than that of the fibre-stabilised microcomb $\left(\sim 3 \times 10^{-12}\right.$ at $\left.40 \mathrm{~s}\right)$.

Finally, the microwave phase noise of the fibre-stabilised microcomb is compared with that of previous microcombs (operating in the $\mathrm{X}-$-, Ku- and $\mathrm{K}$-bands) in Fig. 4, where all phase noise PSDs are scaled to the same $22-\mathrm{GHz}$ carrier frequency.

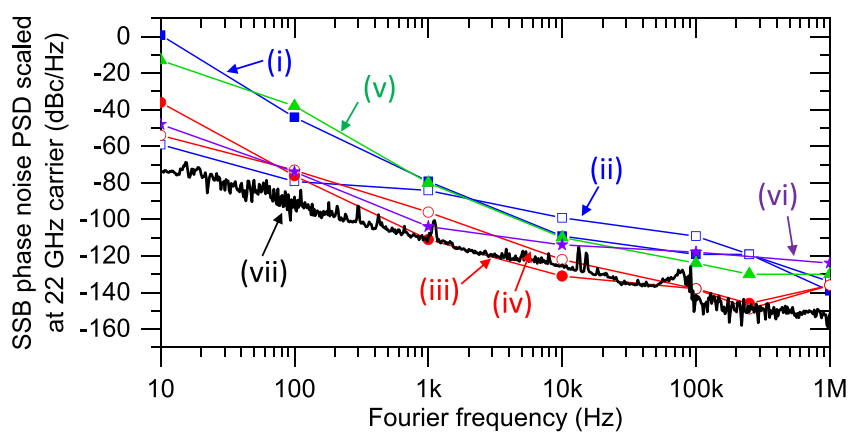

Fig. 4 Phase noise performance comparison. Phase noise comparison with other microcomb-based microwaves scaled to $22 \mathrm{GHz}$. (i) Free-running 20$\mathrm{GHz} \mathrm{Si}_{3} \mathrm{~N}_{4}$ microcomb 33 , (ii) Stabilised $20-\mathrm{GHz} \mathrm{Si}_{3} \mathrm{~N}_{4}$ microcomb ${ }^{33}$, (iii) Free-running 14- $\mathrm{GHz} \mathrm{MgF}_{2}$ microcomb ${ }^{32}$, (iv) Stabilised 14-GHz $\mathrm{MgF}_{2}$ microcomb 32 , (v) Free-running 22- $\mathrm{GHz} \mathrm{SiO}_{2}$ microcomb $^{31}$, (vi) Freerunning $10-\mathrm{GHz} \mathrm{MgF}_{2}$ microcomb ${ }^{60}$. (vii) Fibre-stabilised 22- $\mathrm{GHz} \mathrm{SiO}_{2}$ microcomb (this work). Source data for Fig. 4 are provided in the Supplementary Information. 
Overall, our result exhibits lower phase noise compared to other material platforms (bulk crystals, silica and silicon nitrides) $)^{31-33,60}$ as well as microwave source-stabilised combs ${ }^{32,33}$.

\section{Discussion}

In summary, we have generated both a low-jitter soliton-pulse train and low-noise K-band microwave signal from a compact photonic platform by combining an ultralow-jitter silica microcomb, an AOFS-based broadband repetition-rate tuning mechanism and an ultrastable fibre delayline. Previously, lowphase-noise microwaves over millisecond time scales from microcombs were obtainable only by using bulky external reference sources such as RF signal generators $32-35,37,38,47$, electrooptic combs ${ }^{61,62}$, cavity-locked CW lasers ${ }^{34,39}$ and fully locked optical frequency combs ${ }^{33,63}$. In this work, we showed that the phase noise and frequency fluctuation of the demonstrated silica microcomb can be effectively suppressed by using fibre-optic components fully packaged in a palm-sized enclosure without a vacuum environment. Due to its compact size and alignment-free operation, the demonstrated system will greatly advance lownoise photonic microwave generators for field applications, including photonics-based radars ${ }^{41}, 5 \mathrm{G} / 6 \mathrm{G}$ telecommunications, software-defined radios ${ }^{64}$, signal analysis and $\mathrm{VLBI}^{14,15}$ : as a specific example, photonics-based radars ${ }^{41}$, which combine optical pulse-based sampling/ ADC and low-noise microwave generation, can be greatly benefited by having both high repetitionrate $(>20 \mathrm{GHz})$ low-jitter optical pulse trains and very low-phase noise K-band microwave signals generated from a compact platform. The demonstrated fibre-photonic stabiliser is also readily applicable to the stabilisation of other microcombs with different material platforms ${ }^{59,65-73}$ and repetition rates reaching even the terahertz range ${ }^{26,62,74}$. We also believe that the system has high potential to become even more compact to the chip scale by combining it with silicon photonics. For example, fibre-Bragg gratings and WDM couplers can be replaced with the chip-scale WDMs, and a fibre link can be replaced with a silicon chip $^{75}$ or a microresonator ${ }^{76}$.

\section{Methods \\ Resonator fabrication. Silica wedge resonators with a radius of $3 \mathrm{~mm}$ are fabri- cated through wet oxidation (Tystar, Tytan Mini 1800), optical lithography (SUSS Micro Tec, MA-6 aligner), wet etching (J.T. Baker, buffered oxide etchant 6:1) and dry etching (Teraleader, $\mathrm{XeF}_{2}$ etching system), as detailed in ref. ${ }^{77}$. A high-purity float-zone silicon wafer with an 8 - $\mu$ m oxide film is used. The difference in repe- tition rate is implemented as a difference in the resonator size (several micro- metres). Since both resonators are fabricated at the same time and share the same geometric structure, the wedge angle is $\sim 16^{\circ}$ and the second-order dispersion parameter $\mathrm{D}_{2} / 2 \pi$ is $13.3 \mathrm{kHz}$. The intrinsic $\mathrm{Q}$ factor of both resonators is 250 million.}

Soliton mode-locking and microcomb. The soliton mode is locked using the power kicking method and active capture technique ${ }^{43,44}$ (Supplementary Fig. 1). A narrow linewidth CW laser is used as the pump laser. The pumping power is $\sim 120 \mathrm{~mW}$. The pump is coupled to a microresonator by a tapered fibre, where the polarisation is adjusted to the microresonator by a polarisation controller. First, the laser frequency is swept from the blue side with a sweep rate of $\sim 1 \mathrm{MHz} / \mu$ s using an arbitrary waveform generator (AWG). As the coupling power to the soliton mode increases, a frequency comb is formed via FWM beyond the threshold for parametric oscillation. After the frequency sweep near the cavity resonance, the pump power is quickly modulated (it decreases for a few $\mu$ s and then increases for $50-150 \mu \mathrm{s}$ ) using an $\mathrm{AOFS}_{1}$, and the soliton is triggered. $\mathrm{AOFS}_{1}$ is driven by a VCO whose working range is $51-59 \mathrm{MHz}$. A laser servo is engaged after the soliton is triggered, and the laser frequency control via the laser PZT achieves the long-term operation of the soliton. Here, the photodetected soliton power is used for the servo error.

High-speed repetition-rate tuning mechanism. In our experiment, $\mathrm{AOFS}_{1}$ in Supplementary Fig. 5 is used for both power kicking and stabilisation. To implement both functions using $\mathrm{AOFS}_{1}$, we customised the voltage-controlled oscillator
(VCO) to modulate the power and frequency. The power kicking and the repetition-rate stabilisation are conducted through the power modulation port and the frequency modulation port of the VCO, respectively. As the frequency of the pump laser is already locked to the specific soliton mode via PZT, we applied the frequency modulation of VCO for the repetition-rate stabilisation. We could implement the stabilisation based on the frequency modulation for broadband feedback control loop over $100 \mathrm{kHz}$ bandwidth.

Fibre delayline-based repetition-rate stabilisation method. For repetition-rate phase noise stabilisation, a Michelson interferometer is introduced (Type 2 in Supplementary Fig. 10). The time delay $(\tau)$ is introduced by a fibre link, which determines the stability of the fibre reference system $(\delta \tau / \tau)$, the frequency discrimination sensitivity (scaling with $\tau$ ) and the achievable locking bandwidth (scaling with $1 / \tau$ ). A compact 1 -km-long fibre (diameter $70 \mathrm{~mm}$, height $18 \mathrm{~mm}$ ) is used for the main experiment. Each arm of the fibre Michelson interferometer is split by a fibre coupler. Faraday rotating mirrors are used as end mirrors of the interferometer. An $\mathrm{AOFS}_{2}$ driven by $f_{A O}$ is inserted to perform the synchronous detection to reduce background noise. At the interferometer output, each frequency mode is filtered by a WDM and photodetected. The phase noise of the photodetected signal at $2 f_{A O}$ (due to the round-trip in the interferometer) contains the frequency noise of each filtered mode weighted by the time delay, in the form of $\delta\left[\tau \times\left(m f_{\text {rep }}+f_{\text {ceo }}+2 f_{\text {AO }}\right)\right]$ and $\delta\left[\tau \times\left(n f_{\text {rep }}+f_{\text {ceo }}+2 f_{\text {AO }}\right)\right]$. Each photodetected signal is filtered by an RF-bandpass filter at $2 f_{\mathrm{AO}}$, amplified and down-converted to the baseband by a frequency mixer. The lowpass-filtered baseband mixer output contains the frequency noise of $\delta\left[\tau \times(m-n) f_{\text {rep }}\right]$ by rejecting common-mode $f_{\text {ceo }}$ noise and $2 f_{\mathrm{AO}}$ noise. Note that, in this experiment, $(m-n) f_{\text {rep }}$ corresponds to $\sim 2.5 \mathrm{THz}$ due to the frequency difference between $m f_{\text {rep }}+f_{\text {ceo }}$ and $n f_{\text {rep }}+f_{\text {ceo }}$. Since the pump laser PZT is already used to prevent the soliton from experiencing thermal destabilization, the phase noise is suppressed via the extra frequency modulation of the pump frequency using the VCO applied to the AOFS (AOFS 1 in Fig. 1 and Supplementary Fig. 5).

\section{Data availability}

Source data are provided with this paper.

\section{Code availability}

The simulation and computational codes for this study are available from the corresponding authors on request.

Received: 2 April 2021; Accepted: 15 December 2021; Published online: 19 January 2022

\section{References}

1. Yao, X. S. \& Maleki, L. High frequency optical subcarrier generator. Electron. Lett 30, 1525-1526 (1994).

2. Matsko, A., Maleki, L., Savchenkov, A. A. \& Ilchenko, V. S. Whispering gallery mode based optoelectronic microwave oscillator. J. Mod. Opt. 50, 2523-2542 (2003).

3. Li, J., Yi, X., Lee, H., Diddams, S. A. \& Vahala, K. J. Electro-optical frequency division and stable microwave synthesis. Science 345, 309-313 (2014).

4. Li, J., Lee, H. \& Vahala, K. Microwave synthesizer using an on-chip Brillouin oscillator. Nat. Commun. 4, 2097 (2013)

5. Li, J. \& Vahala, K. Ultra-low phase-noise microwave oscillator based on electro-optical frequency division. In 2017 Joint Conference of the European Frequency and Time Forum and IEEE International Frequency Control Symposium (EFTF/IFCS) 265-266 (IEEE, 2017).

6. Kalubovilage, $M$. et al. Ultra-low phase noise microwave generation with a free-running monolithic femtosecond laser. Opt. Express 28, 25400-25409 (2020).

7. Bartels, A. et al. Femtosecond-laser-based synthesis of ultrastable microwave signals from optical frequency references. Opt. Lett. 30, 667-669 (2005).

8. McFerran, J. J. et al. Low noise synthesis of microwave signals from an optical source. Electron. Lett. 41, 36-37 (2005).

9. Fortier, T. M. et al. Generation of ultrastable microwaves via optical frequency division. Nat. Photonics 5, 425-429 (2011).

10. Xie, X. et al. Photonic microwave signals with zeptosecond-level absolute timing noise. Nat. Photonics 11, 44-47 (2017).

11. Kim, J., Cox, J. A., Chen, J. \& Kärtner, F. X. Drift-free femtosecond timing synchronization of remote optical and microwave sources. Nat. Photonics 2 , 733-736 (2008).

12. Schulz, S. et al. Femtosecond all-optical synchronization of an X-ray freeelectron laser. Nat. Commun. 6, 5938 (2015).

13. Xin, M. et al. Attosecond precision multi-kilometer laser-microwave network. Light Sci. Appl. 6, e16187 (2017). 
14. Clivati, C. et al. A VLBI experiment using a remote atomic clock via a coherent fibre link. Sci. Rep. 7, 40992 (2017).

15. Pizzocaro, M. et al. Intercontinental comparison of optical atomic clocks through very long baseline interferometry. Nat. Phys. 17, 223-227 (2021).

16. Del'Haye, P. et al. Optical frequency comb generation from a monolithic microresonator. Nature 450, 1214-1217 (2007).

17. Levy, J. et al. CMOS-compatible multiple-wavelength oscillator for on-chip optical interconnects. Nat. Photonics 4, 37-40 (2010).

18. Razzari, L. et al. CMOS-compatible integrated optical hyper-parametric oscillator. Nat. Photonics 4, 41-45 (2010).

19. Savchenkov, A. A. et al. Low threshold optical oscillations in a whispering gallery mode $\mathrm{CaF}_{2}$ resonator. Phys. Rev. Lett. 93, 243905 (2004).

20. Li, J., Lee, H., Chen, T. \& Vahala, K. J. Low-pump-power, low-phase-noise, and microwave to millimeter-wave repetition rate operation in microcombs Phys. Rev. Lett. 109, 233901 (2012).

21. Gaeta, A. L., Lipson, M. \& Kippenberg, T. J. Photonic-chip-based frequency combs. Nat. Photonics 13, 158-169 (2019).

22. Diddams, S. A., Vahala, K. \& Udem, T. Optical frequency combs: Coherently uniting the electromagnetic spectrum. Science 369, eaay3676 (2020).

23. Matsko, A. B. \& Maleki, L. On timing jitter of mode locked Kerr frequency combs. Opt. Express 21, 28862-28876 (2013).

24. Jeong, D. et al. Ultralow jitter silica microcomb. Optica 7, 1108-1111 (2020).

25. Bao, C. et al. Quantum diffusion of microcavity solitons. Nat. Phys. 17, 462-466 (2021).

26. Spencer, D. T. et al. An optical-frequency synthesizer using integrated photonics. Nature 557, 81-85 (2018).

27. Suh, M.-G., Yang, Q.-F., Yang, K. Y., Yi, X. \& Vahala, K. J. Microresonator soliton dual-comb spectroscopy. Science 354, 600-603 (2016).

28. Trocha, P. et al. Ultrafast optical ranging using microresonator soliton frequency combs. Science 359, 887-891 (2018).

29. Riemensberger, J. et al. Massively parallel coherent laser ranging using a soliton microcomb. Nature 581, 164-170 (2020).

30. Obrzud, E. et al. A microphotonic astrocomb. Nat. Photonics 13, 31-35 (2019).

31. Yi, X. et al. Single-mode dispersive waves and soliton microcomb dynamics. Nat. Commun. 8, 14869 (2017).

32. Weng, W. et al. Spectral purification of microwave signals with disciplined dissipative Kerr solitons. Phys. Rev. Lett. 122, 013902 (2019).

33. Liu, J. et al. Photonic microwave generation in the X- and K-band using integrated soliton microcombs. Nat. Photonics 14, 486-491 (2020).

34. Lucas, E. et al. Ultralow-noise photonic microwave synthesis using a soliton microcomb-based transfer oscillator. Nat. Commun. 11, 374 (2020).

35. Yang, Q. F. et al. Dispersive-wave induced noise limits in miniature soliton microwave sources. Nat. Commun. 12, 1442 (2021).

36. Seidel, D., Savchenkov, A., Eliyahu, D., Williams, S. \& Matsko, A. Minituarized Ka-band photonic oscillator. In 2018 International Topical Meeting on Microwave Photonics (MWP) 1-2 (IEEE, 2018).

37. Del'Haye, P. et al. Phase-coherent microwave-to-optical link with a selfreferenced microcomb. Nat. Photonics 10, 516-520 (2016).

38. Stern, L. et al. Direct Kerr frequency comb atomic spectroscopy and stabilization. Sci. Adv. 6, eaax6230 (2020).

39. Lamb, E. S. et al. Optical-frequency measurements with a Kerr microcomb and photonic-chip supercontinuum. Phys. Rev. Appl. 9, 024030 (2018).

40. Kwon, D. et al. Generation of multiple ultrastable optical frequency combs from an all-fiber photonic platform. Sci. Adv. 6, eaax4457 (2020).

41. Ghelfi, P. et al. A fully photonics-based coherent radar system. Nature 507, 341-345 (2014).

42. Jung, K. \& Kim, J. All-fibre photonic signal generator for attosecond timing and ultralow-noise microwave. Sci. Rep. 5, 16250 (2015).

43. Yi, X. et al. Soliton frequency comb at microwave rates in a high-Q silica microresonator. Optica 2, 1078-1085 (2015).

44. Herr, T. et al. Temporal solitons in optical microresonators. Nat. Photonics 8 , 145-152 (2014).

45. Kwon, D. et al. Reference-free, high resolution measurement method of timing jitter spectra of optical frequency combs. Sci. Rep. 7, 40917 (2017).

46. Gorodetsky, M. L. \& Grudinin, I. S. Fundamental thermal fluctuations in microspheres. J. Opt. Soc. Am. B 21, 697-705 (2004).

47. Stone, J. R. et al. Thermal and nonlinear dissipative-soliton dynamics in Kerr-microresonator frequency combs. Phys. Rev. Lett. 121, 063902 (2018).

48. Yu, B.-M. et al. Single-chip Si optical single-sideband modulator. Photonics Res. 6, 6-11 (2018).

49. Komljenovic, T. et al. Heterogeneous silicon photonic integrated circuits. J. Lightwave Technol. 34, 20-35 (2016).

50. Jin, W. et al. Hertz-linewidth semiconductor lasers using CMOS-ready ultrahigh-Q microresonators. Nat. Photonics 15, 346-353 (2021).

51. Shen, B. et al. Integrated turnkey soliton microcombs. Nature 582, 365-369 (2020).
52. Xie, X. et al. Effect of pulse duration fluctuation on the photodetection of a train of pulses from an optical frequency comb. In 2018 European Frequency and Time Forum (EFTF) 318-321 (IEEE, 2018).

53. Duan, L. General treatment of the thermal noises in optical fibers. Phys. Rev. A 86, 023817 (2012)

54. Cahill, J. P., Okusaga, O., Zhou, W., Menyuk, C. R. \& Carter, G. M. Superlinear growth of Rayleigh scattering-induced intensity noise in singlemode fibers. Opt. Express 23, 6400-6407 (2015).

55. Nelson, C. W. \& Howe, D. A. A sub-sampling digital PM/AM noise measurement system. In Proc. IEEE Frequency Control Symposium. 1-4 (IEEE, 2012).

56. Riley, W. J. Handbook of Frequency Stability Analysis (NIST, 2008).

57. Świerad, D. et al. Ultra-stable clock laser system development towards space applications. Sci. Rep. 6, 33973 (2016).

58. Gray, D. E. American Institute of Physics Handbook (McGraw-Hill, 1963).

59. Bousonville, M. et al. New phase stable optical fiber. In Proc. Beam Instrumentation Workshop 2012 (BIW2012) 101-103 (JACoW, 2012).

60. Liang, W. et al. High spectral purity Kerr frequency comb radio frequency photonic oscillator. Nat. Commun. 6, 7957 (2015).

61. Liu, J. et al. Monolithic piezoelectric control of soliton microcombs. Nature 583, 385-390 (2020).

62. Tetsumoto, T. et al. $300 \mathrm{GHz}$ wave generation based on a Kerr microresonator frequency comb stabilized to a low noise microwave reference. Opt. Lett. 45, 4377-4380 (2020).

63. Del'Haye, P. et al. Full stabilization of a microresonator-based optical frequency comb. Phys. Rev. Lett. 101, 053903 (2008).

64. Abidi, A. A. The path to the software-defined radio receiver. IEEE J. SolidState Circuits 42, 954-966 (2007).

65. Obrzud, E., Lecomte, S. \& Herr, T. Temporal solitons in microresonators driven by optical pulses. Nat. Photonics 11, 600-607 (2017).

66. Webb, K. E., Erkintalo, M., Coen, S. \& Murdoch, S. G. Experimental observation of coherent cavity soliton frequency combs in silica microspheres. Opt. Lett. 41, 4613-4616 (2016).

67. Stern, B. et al. Battery-operated integrated frequency comb generator. Nature 562, 401-405 (2018).

68. Brasch, V. et al. Photonic chip-based optical frequency comb using soliton Cherenkov radiation. Science 351, 357-360 (2016).

69. Jia, K. et al. Photonic flywheel in a monolithic fiber resonator. Phys. Rev. Lett. 125, 143902 (2020).

70. Chang, L. et al. Ultra-efficient frequency comb generation in AlGaAs-oninsulator microresonators. Nat. Commun. 11, 1331 (2020).

71. Jung, H., Xiong, C., Fong, K. Y., Zhang, X. \& Tang, H. X. Optical frequency comb generation from aluminum nitride microring resonator. Opt. Lett. 38, 2810-2813 (2013).

72. He, Y. et al. Self-starting bi-chromatic $\mathrm{LiNbO}_{3}$ soliton microcomb. Optica 6, 1138-1144 (2019)

73. Gong, Z., Liu, X., Xu, Y. \& Tang, H. Near-octave lithium niobate soliton microcomb. Optica 7, 1275-1278 (2020).

74. Kuse, N., Briles, T. C., Papp, S. B. \& Fermann, M. E. Control of Kerrmicroresonator optical frequency comb by a dual-parallel Mach-Zehnder interferometer. Opt. Express 27, 3873-3883 (2019).

75. Lee, H. et al. Ultra-low-loss optical delay line on a silicon chip. Nat. Commun. 3, 867 (2012)

76. Alnis, J. et al. Thermal-noise-limited crystalline whispering-gallery-mode resonator for laser stabilization. Phys. Rev. A 84, 011804 (2011).

77. Lee, $H$. et al. Chemically etched ultrahigh-Q wedge-resonator on a silicon chip. Nat. Photonics 6, 369-373 (2012).

\section{Acknowledgements}

This research was supported by the Institute for Information and Communications Technology Promotion of Korea (Grant no. 2019-0-01349 for JK and HL) and the National Research Foundation of Korea (Grant no. 2021R1A2B5B03001407 for JK). We thank Minji Hyun for the discussions. We also thank the Center for Time and Frequency, Korea Research Institute of Standards and Science (KRISS), for loaning the phase noise analyser.

\section{Author contributions}

D.K. and J.K. conceived the idea and designed the experiment. J.K. and H.L. supervised the project. D.J. and H.L. designed and fabricated the silica microresonator. D.K., D.J. and I.J. performed the experiment and obtained data. D.K. and J.K. analysed the data and wrote the manuscript with inputs from all authors.

\section{Competing interests}

The authors declare no competing interests. 


\section{Additional information}

Supplementary information The online version contains supplementary material available at https://doi.org/10.1038/s41467-022-27992-8.

Correspondence and requests for materials should be addressed to Hansuek Lee or Jungwon Kim.

Peer review information Nature Communications thanks Qi-Fan Yang, Olivier Llopis and the anonymous reviewer(s) for their contribution to the peer review of this work Peer reviewer reports are available.

Reprints and permission information is available at http://www.nature.com/reprints

Publisher's note Springer Nature remains neutral with regard to jurisdictional claims in published maps and institutional affiliations. (c) (P) Open Access This article is licensed under a Creative Commons Attribution 4.0 International License, which permits use, sharing, adaptation, distribution and reproduction in any medium or format, as long as you give appropriate credit to the original author(s) and the source, provide a link to the Creative Commons license, and indicate if changes were made. The images or other third party material in this article are included in the article's Creative Commons license, unless indicated otherwise in a credit line to the material. If material is not included in the article's Creative Commons license and your intended use is not permitted by statutory regulation or exceeds the permitted use, you will need to obtain permission directly from the copyright holder. To view a copy of this license, visit http://creativecommons.org/ licenses/by/4.0/.

(C) The Author(s) 2022 